\title{
Reflex sympathetic dystrophy/complex regional pain syndrome, type 1
}

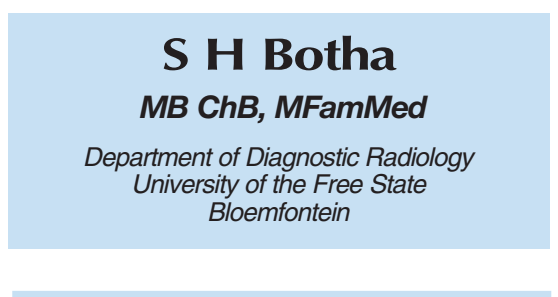

\section{Abstract}

Complex regional pain syndrome (CPRS), type 1 is a pain disorder that develops unpredictably and can follow a minor injury. A 12-year-old boy presented with severe pain in the feet and could not walk or stand weight bearing. Normal X-rays showed osteopenic changes and radiolucent lines, which appeared to be stress fractures. Three-phase bone scintigraphy showed no uptake in the left lower leg on the blood pool phase or on the immediate or delayed images. This indicated typical CPRS type 1 in children. The uptake in the right foot was increased and the stress fracture and other illness could not be differentiated. Computed tomography was done to exclude stress fractures. Only osteopenic changes in both calcaneus bones were found and there was no evidence of cortical stress fractures. Magnetic resonance images revealed oedema in the calcaneus and talus bones of both feet. The patient received epidural narcotic infusion with sympathetic blockage for 1 week combined with extensive physiothera- py. The blood pool phase of the bone scan became normal within 2 weeks, and increased uptake in both feet was noticed. The patient was followed up with MRI every 3 months and the bone marrow oedema disappeared after 6 months.

\section{Introduction}

Reflex sympathetic dystrophy (RSD) or the newer synonym, complex regional pain syndrome (CPRS), type 1 is a pain disorder that develops unpredictably and can follow a minor injury. ${ }^{1,2}$ CRPS type 1 is not limited to a single peripheral nerve distribution, and is associated with oedema, changes in skin blood flow, abnormal sudomotor activity in the region of the pain, and allodynia or hyperalgesia. In CRPS type 2 or causalgia there is history of a peripheral nerve injury, thus the pain and autonomic disturbance can be identical to CRPS type 1 but has a nerve-specific distribution. ${ }^{2}$

\section{Case report}

A 12-year-old boy presented with severe pain in the feet, worse in the left foot, which increased over a period of 2 weeks to such an extent that he could not walk or stand weight bearing. Although he was active in a number of sports no incident of injury was reported.
On examination both feet were cold, pale and mildly swollen and he experienced paraesthesiae (pins and needles), hyperalgesia (extreme pain response to a minimally painful stimulus) and allodynia (unpleasant painful sensation induced by nonpainful stimulus such as light touch on the affected area).

Normal X-rays were taken of both feet. These showed marked osteopenic changes and radiolucent lines which appeared to be stress fractures, especially in the left calcaneus (Figs 1a and $1 \mathrm{~b}$ and Figs $2 \mathrm{a}$ and $2 \mathrm{~b}$ ).

Three-phase bone scintigraphy was done, which showed no uptake in the left lower leg on the blood pool

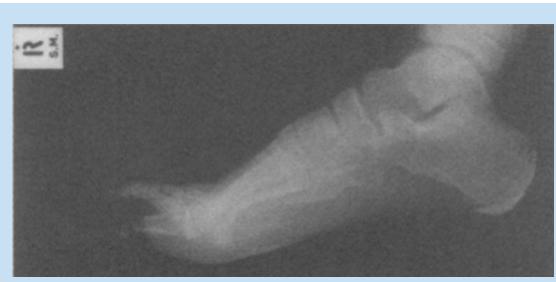

Fig. 1a. Lateral $X$-ray of right foot showing radiolucent lines and osteopenia.

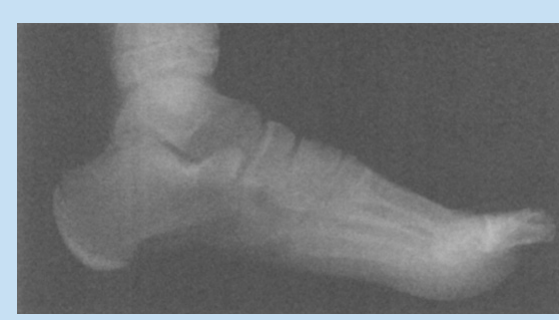

Fig.1b. Lateral X-ray of left foot showing radiolucent lines and osteopenia.

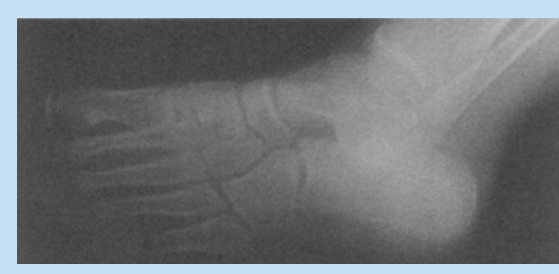

Fig. 2a. Oblique $X$-rays of right foot showing possible stress fractures of calcaneus bones. 


\section{CASE REPORTS}

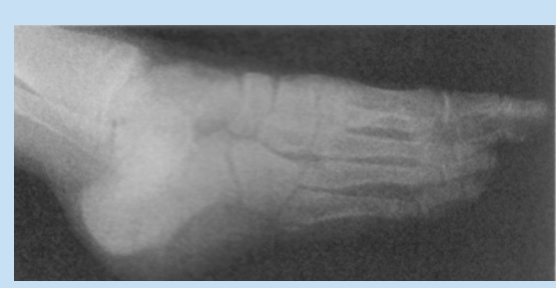

Fig. 2b. Oblique X-rays of left foot showing possible stress fractures of calcaneus bones.

phase or on the immediate or delayed images (Fig. 3). This indicated typical RSD in children. In contrast, adult uptake is usually increased. When the intensity was increased, the delayed images in the left calcaneus showed a slight increase in uptake. The uptake in the right foot was increased and the stress fracture and other illness could not be differentiated (Fig. 4).

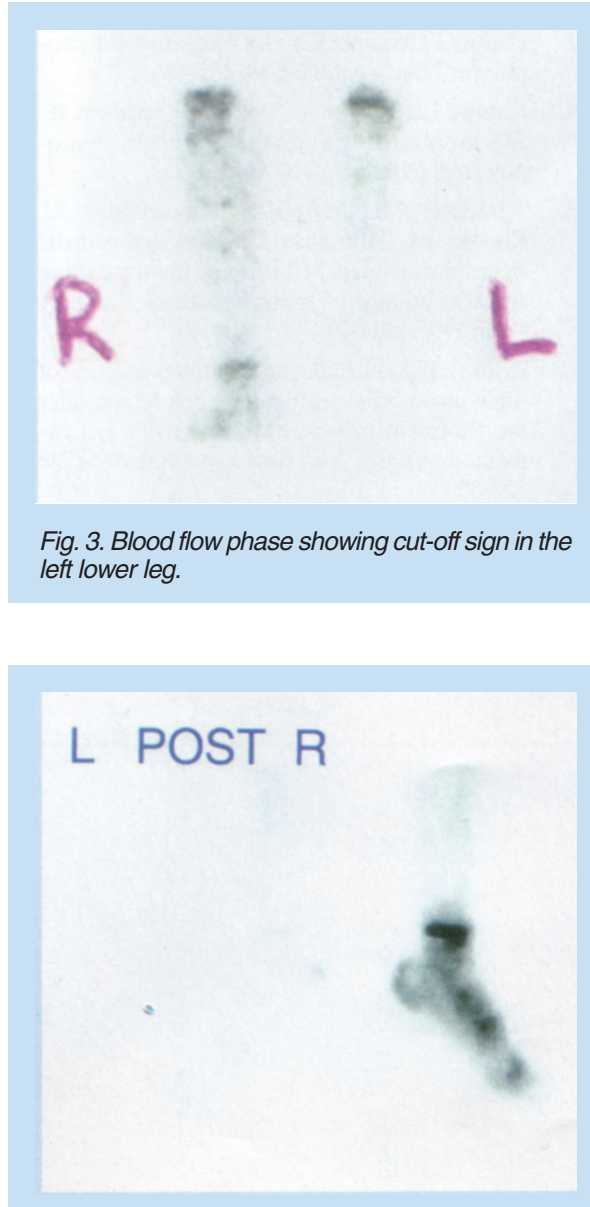

Fig. 4. Blood pool phase showing still no uptake in the left leg with increased uptake in the right foot.
Computed tomography (CT) was done to exclude stress fractures. Only osteopenic changes in both calcaneus bones were found and there was no evidence of cortical stress fractures (Fig. 5).

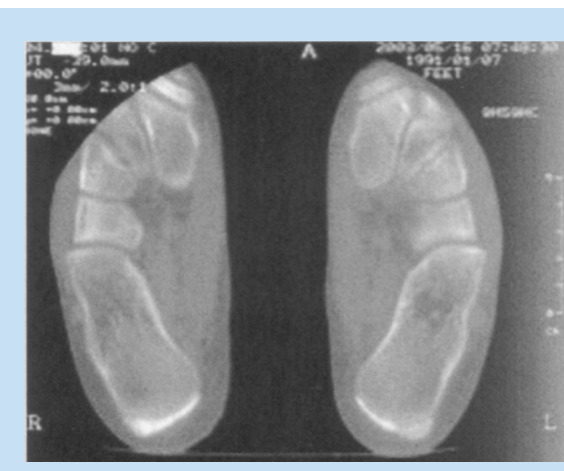

Fig. 5. Axial CT image of both feet showing osteopenic changes.

Magnetic resonance images (MRIs) were taken of both feet and revealed marked oedema in the calcaneus and talus bones of both feet. Some soft-tissue oedema especially over the left ankle was also present (Fig. 6 and Figs $7 \mathrm{a}$ and $7 \mathrm{~b}$ ).

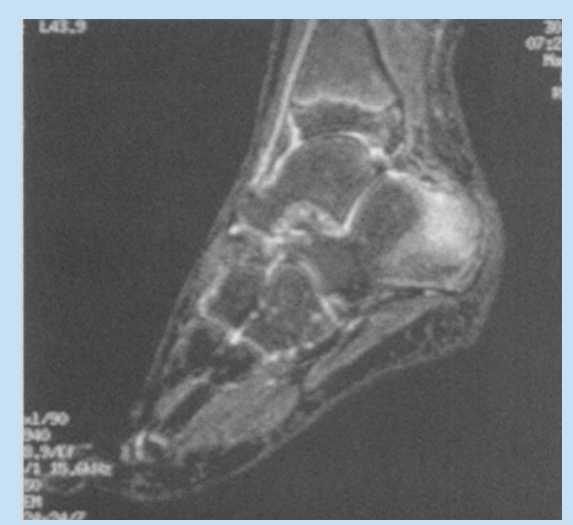

Fig. 6. Sagittal STIR image of the left foot showing soft-tissue oedema and bone marrow oedema in calcaneus and talus bones.

There was contradiction between the two diagnoses of stress fractures and CPRS type 1 . As CPRS type 1 is a clinical diagnosis the boy was treated as such. He was admitted into hospital and received epidural narcotic infusion

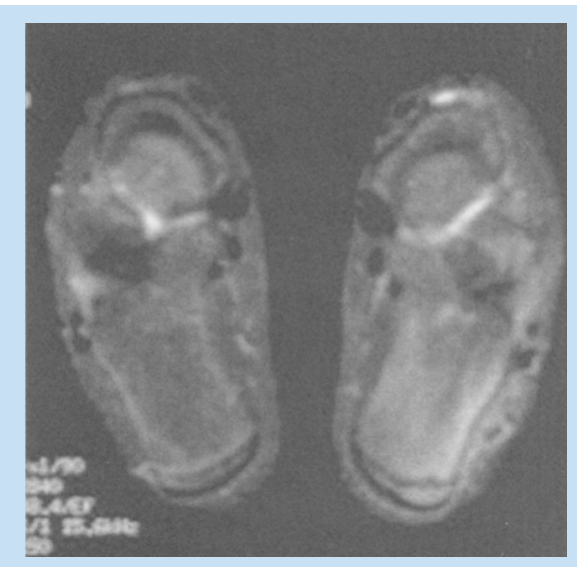

Fig. 7a. Axial STIR MR images of both feet showing oedema of soft tissue and bone marrow.

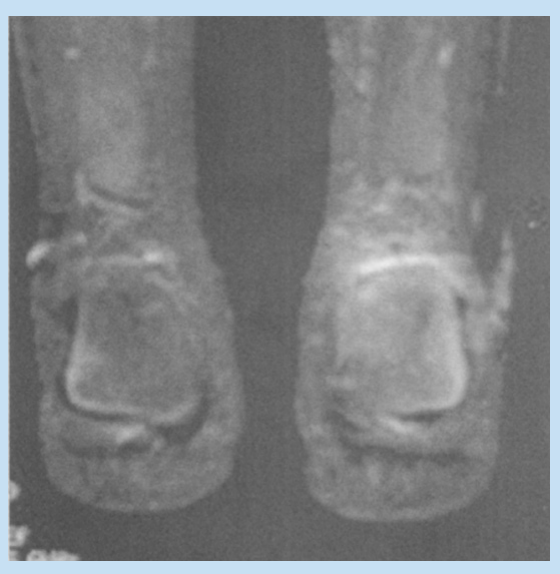

Fig. 7b. Coronal STIR MR images of both feet showing oedema of soft tissue and bone marrow.

with sympathetic blockage for a period of 1 week combined with extensive physiotherapy (3 times a day). Fortunately he recovered remarkably. If he had had stress fractures his condition would have worsened. The blood pool phase of the bone scan became normal within 2 weeks, and increased uptake in both feet was noticed. He was followed up with MRI every 3 months and the bone marrow oedema disappeared after 6 months.

\section{Discussion}

The physiology, diagnosis and management of CPRS type 1 is discussed below. 


\section{Pathophysiology}

In patients with sympathetically maintained pain it is likely that the major site of abnormality is in the wide-dynamic-range neurons in the spinal cord. The hypothesis is that an initiating trauma event sensitises these neurons with associated proliferation of alpha-receptors on low threshold mechanoreceptors and in the surrounding tissues. A normal mechanical stimulus results then in excessive firing of wide-dynamic-range neurons, causing abnormal pain sensation. The proliferation of alpha-receptors in the surrounding tissues, including the blood vessels and other end organs explains the clinical features of autonomic activation seen in the disorder. ${ }^{3}$

\section{Clinical features}

Pain is the main clinical manifestation of CRPS type 1 and is disproportionate to that expected of the severity of the underlying injury. The pain combines with autonomic disturbance such as skin colour, skin temperature, sudomotor activity and swelling changes. ${ }^{1,3}$

\section{Precipitating factors}

The initiating cause in CRPS type 1 may be either trauma of external origin or iatrogenic, post surgery. In some patients particularly children, no definite injury can be identified. ${ }^{1,4}$

\section{Diagnosis}

The diagnosis of CRPS type 1 is essentially clinical and is made on the basis of the combination of excessive pain together with autonomic disturbance in the area of the pain.

\section{Specific investigations}

Plain X-ray: Routine radiography was first used to confirm the presence of CRPS type 1. However, the soft tissue swelling and demineralisation are nonspecific and a late manifestation of the illness. ${ }^{5}$

Bone scan: Bone scintigraphy provides a non-invasive method of diagnosis. In adults it demonstrates increased uptake on both blood pool and delayed images, and in children a decreased uptake in all the phases. This is, however, only $60 \%$ sensitive.,

Magnetic resonance imaging: MRI can provide supportive evidence for CRPS type 1. Soft-tissue changes and bone marrow oedema are early changes and muscle atrophy is present in the disorder's later stages. MRI may also be helpful in patient follow-up.

\section{Management}

The treatment of CRPS type 1 includes physiotherapy, drugs (analgesia, antidepressants, anticonvulsants) and sympathetic blockade. ${ }^{1,3}$

\section{Conclusion}

Although CPRS type 1 is a clinical diagnosis, imaging methods such as bone scintigraphy and MRI are helpful in the differential diagnosis and in follow-up of these patients.

\section{References}

1. Blombery PA. A review of reflex sympathetic dystrophy. Aust Fam Physician 1995; 24: 16511655.

2. Walker SM, Cousins MJ. Complex regional pain syndromes: including 'reflex sympathetic dystrophy' and 'causalgia'. Anaesth Intensive Care 1997; 25: $113-125$

3. Phelps GR, Wilentz S. Reflex sympathetic dystrophy. Int J Dermatol 2000; 39: 481-486.

4. Murray CS, Cohen A, Perkins T, Davidson JE, Sills JA. Morbidity in reflex sympathetic dystrophy. Arch Dis Child 2000; 82: 231-233.

5. Schweitzer ME, Mandel S, Schwartzman RJ, Knobler RL, Tahmoush AJ. Reflex sympathetic dystrophy revisited: MR imaging findings before and after infusion of contrast material. Radiology 1995; 195(1):211-214.

6. Zyluk A, Birkenfeld B. Quantitative evaluation of three-phase bone scintigraphy before and after the treatment of post-traumatic reflex sympathetic dystrophy. Nucl Med Commun 1999; 20: 327-333. 